\title{
Ground state lifetime of strong-coupled polaron in an asymmetric quantum dot
}

\author{
Zhi-Xin Li ${ }^{a, *}$ and Jing-Lin Xiao ${ }^{b}$ \\ ${ }^{a}$ College of Physics and Chemical, Hebei Normal University of Science and \\ Technology, Qinhuangdao, Hebei 066004, China \\ ${ }^{b}$ College of Physics and Electronic Information, Inner Mongolia Normal University, \\ Tongliao 028043, China
}

Received 25 June 2010; Accepted (in revised version) 28 July 2010

Published online 17 January 2011

\begin{abstract}
On the condition of electron and LO-phonons strong-coupled, the ground-state energy of polaron has been obtained by using linear combination operator and unitary transformation methods in an asymmetric quantum dot. Quantum transition which causes the changes of the polaron lifetime is occurred in the quantum system due to the electronphonon interaction and the influence of external temperature effect which is the polaron leap from the ground-state to the first-excited state absorbing a LO-phonon. Numerical calculation is performed and the results show that the ground-state lifetime of polaron increases with increasing the ground-state energy and decreases with increasing the couplingstrength. The ground-state lifetime is extented with the shorten of the temperature. It is also observed that the ground-state lifetime is a decreasing function of the transverse and longitudinal confinement lengths of the quantum dot.
\end{abstract}

PACS: 73.21.La, 03.67.Ac, 85.35.Be

Key words: asymmetric quantum dot, strong-coupled, ground-state lifetime, polaron

\section{Introduction}

Recently, with the rapid development of nanofabrication technology of materials, quantum dot, quantum well and quantum wire, low dimensional electronic systems have been easily produced with molecular beam extension (MBE) technique, metal organic chemical vapor deposition (MOCVD) and chemical self-assemble technique. Due to the small structures of the quantum dot, some physical properties such as optical and electron transport characteristics are quite different from those of the bulk materials. More and more physicists worldwide are

${ }^{*}$ Corresponding author. Email address: zzlxx2006@126.com (Z. X. Li) 
paying considerable attention to the research of the local electron in a quantum dot (QD) by many theoretically [1-3] and experimentally [4-6] methods.

In recent years, the most feasible approach has been quantum dots for realizing the quantum computer because of the advantage of being integrated. The two quantum state of electron is employed as a single qubit in a quantum dot [7-9]. In practice, the electron-phonon interaction is essential to understand the optical absorption spectra in semiconductors. Research on the polaron effect has become a main subject in the physics of low-dimensional quantum systems. Especially in a quantum dot system, the electron-phonon interactions are enhanced by the geometric confinement. Therefore, a number of studies have been focused on the influence of the electron-phonon interactions on polaron in parabolic quantum dots.

Xie and Chen [10] studied the thickness dependence of the binding energy of an impurity bound polaron in a parabolic QD in magnetic fields by using the second-order perturbation theory. Au-Yeung et al. [11] investigated the combined effects of a parabolic potential and a Coulomb impurity on the cyclotron resonance of a three-dimensional magnetopolaron by using Larsen's perturbation method, under the condition of strong parabolic potential. Within the framework of Femman-haken path integral theory, Ren et al. [12], calculated the ground state energy of two-dimension polarons in asymmetric quantum dots for arbitrary electronphonon coupling strengths. Employing the frame work of Feynman variational path integral theory, Chen et al. [13] derived an expression of the ground state energy of an electron coupled simultaneously with a coulomb potential and a longitudinal-optical phonon field in parabolic asymmetric quantum dots and quantum wires. The properties of strong-coupling bound magnetopolaron of quantum dot in an asymmetry confinement potential have been studied using Pekar's varitional method by Chen and Xiao [14]. Wang et al. [15] recently studied the binding energy of hydrogenised impurities in a GaAs cylindrical QD using a twoparameter variational wave function. By introducing a trail wave function constructed as a direct form of an electronic part and a part of coherent phonons. Kandemir and Cetin [16] investigated the polaronic effect on the low-lying energy levels of an electron bound to a hydrogenic impurity in a three-dimensional anisotropic harmonic potential subjected to a uniform magnetic field. Boucaud et al. [17] studied the polaron decay in InAs/GaAs selfassembled quantum dots by pump-probe spectroscopy and Zibik et al. [18] investigated the polaron decay in n-type InAs quantum dots using energy dependent, mid-infrared pump-probe spectroscopy, However, the ground-state lifetime of polaron in a parabolic QD has not been investigated so far in these works.

In this paper, the properties of the ground-state lifetime of polaron in an asymmetric quantum dot will be studied by using linear combination operator and unitary transformation methods. The effect of the ground-state energy, electron-phonons coupling-strength, external temperature and the transverse and longitudinal confinement lengths of quantum dot on the ground state lifetime will be discussed. 


\section{Theory}

The electron moves in the polar crystal quantum dot. Due to the phonon field and the polar crystal boundary effects, the motion of the electron in every direction has been quantified. Assuming that the electron in the first quantum dot is bounded by different parabolic potential in the $\mathbf{x}-\mathbf{y}$ plane and the $\mathbf{z}$ direction, the electron-phonon system Hamiltonian in an asymmetric quantum dot can be written as

$$
H=\frac{p^{2}}{2 m^{*}}+\frac{1}{2} m^{*} \omega_{1}^{2} \rho^{2}+\frac{1}{2} m^{*} \omega_{2}^{2} z^{2}+\sum_{w} h \omega a_{w}^{+} a_{w}+\sum_{w}\left(V_{w} e^{i w r} a_{w}+h \cdot c\right),
$$

where $m^{*}$ is the band mass of electron, $\omega_{1}$ and $\omega_{2}$ is the creation (annihilation) operator of the bulk-LO-phonons with wave operator $w, r=(\rho, z)$ is the position vector of an electron,

$$
\begin{aligned}
& V_{w}=i\left(\frac{h \omega_{L O}}{w}\right)\left(\frac{h}{2 m^{*} \omega_{L O}}\right)^{1 / 4}\left(\frac{4 \pi \alpha}{V}\right)^{1 / 2}, \\
& \alpha=\left(\frac{e^{2}}{2 h \omega_{L O}}\right)\left(\frac{2 m^{*} \omega_{L O}}{h}\right)\left(\frac{1}{\varepsilon_{\infty}}-\frac{1}{\varepsilon_{0}}\right) .
\end{aligned}
$$

Following Huybrechts [19], we introduce the linear combination of the creation and annihilation

$$
\begin{aligned}
& p_{j}=\left(\frac{m^{*} \hbar \lambda}{2}\right)\left(b_{j}+b_{j}^{+}\right), \\
& r_{j}=i\left(\frac{\hbar}{2 m^{*} \lambda}\right)^{1 / 2}\left(b_{j}-b_{j}^{+}\right), \quad(j=x, y, z),
\end{aligned}
$$

and carry out unitary transformation

$$
U=\exp \left(\sum_{w}\left(a_{w}^{+} f_{w}-a_{w} f_{w}^{*}\right)\right)
$$

where $\lambda$ and $f_{w}\left(f_{w}^{*}\right)$ are the variational parameters that will be subsequently chosen by minimizing the energy. Then the transformed Hamiltonian can be expressed as

$$
H^{\prime}=U^{-1} H U \text {. }
$$

We choose the following ground state wave function

$$
|\psi\rangle=|0\rangle_{a}|0\rangle_{b}
$$

$|0\rangle_{a}$ is the zero-phonon state, $|0\rangle_{b}$ is the vacuum state of $b$ operator. The expectation value of Eq. (6) with respect to $|\psi\rangle$ can be expressed as $F\left(\lambda, f_{q}\right)=\langle\psi|\grave{H}| \psi\rangle$. Using the variational method, we have

$$
F(\lambda)=\frac{3}{4} h \lambda+\frac{h \omega_{1}^{2}}{2 \lambda}+\frac{h \omega_{2}^{2}}{4 \lambda}-\sum_{w} \frac{\left|V_{w}^{2}\right|}{h \omega_{L}} \exp \left(-\frac{h \omega^{2}}{2 m^{*} \lambda}\right),
$$


where $\lambda$ satisfies

$$
\lambda^{2}-\frac{2}{3} \pi^{1 / 2} \alpha \lambda^{3 / 2}-\left(\frac{2}{3} \omega_{1}^{2}+\frac{1}{3} \omega_{2}^{2}\right)=0 .
$$

By solving Eq. (9), we obtain variational frequency of polaron $\lambda=\lambda_{0}$. Substituting $\lambda_{0}$ into Eq. (8) and choosing in the usual polaron units $\left(h=2 m^{*}=\omega_{L O}=1\right)$, the ground-state energy of strong-coupled polaron in an asymmetric quantum dot can be written as

$$
E_{0}=\frac{3 \lambda_{0}}{4}+\frac{2}{l_{1}^{4} \lambda_{0}}+\frac{1}{l_{2}^{4} \lambda_{0}}-\frac{\alpha}{\sqrt{\pi}} \lambda_{0}^{1 / 2},
$$

where $l_{1}=\sqrt{h / m^{*} \omega_{1}}, l_{2}=\sqrt{h / m^{*} \omega_{2}}$ are the transverse and longitudinal confinement lengths, respectively. Due to electron-phonons interaction and external temperature influences, on the basis of the gold principle, polaron absorbs a LO-phonon, the velocity rate of which transition to the first-excited state from the ground-state can be expressed as

$$
T_{-1}=\frac{\alpha \omega_{L O}}{2 \lambda_{0}} \sqrt{\frac{2 m^{*} \omega_{L O}}{h}} n_{4} \times \ln \frac{\left(\sqrt{\lambda_{0}^{2}+2 m^{*} \omega_{L O} / h}+\lambda_{0}\right)^{2}}{\left(\sqrt{\lambda_{0}^{2}-2 m^{*} \omega_{L O} / h}-\lambda_{0}\right)^{2}}
$$

According to quantum statistics, we have

$$
n_{q}=\left(\exp \left(\frac{h \omega_{L O}}{K_{B} T}\right)-1\right)^{-1},
$$

where $T$ and $\lambda_{0}$ are the ground state lifetime and the ground state wave vector of polaron, respectively, $K_{B}$ stands for Boltzmann constant. The $n_{q}$ and Eq. (12) is self-consistent. We assume that the temperature parameter can be defined as $h \omega_{L O} / K_{B} T=\gamma$. Then Eq. (12) can be rewritten as

$$
n_{q}=(\exp \gamma-1)^{-1}
$$

\section{Results and discussion}

In this section, to better demonstrate the effects of the ground-state energy, the electronphonon coupled-strength, the external temperature and the transverse and longitudinal confinement lengths on the ground-state lifetime, numerical computations will be performed. The results are presented in Figs. 1-4.

Fig. 1 shows the relationship between the ground-state lifetime and the ground-state energy, at $\gamma=0.55,0.6,0.7$. From the Fig. 1, it can be seen that the ground-state lifetime increases by increasing the ground-state energy of polaron. That is because the higher of the ground-state energy of polaron, the lower the transiton probability per second, which is a measure of how quikly the polaron is scattered from its ground-state into the first-excited after absorbing a LO-phonon. 


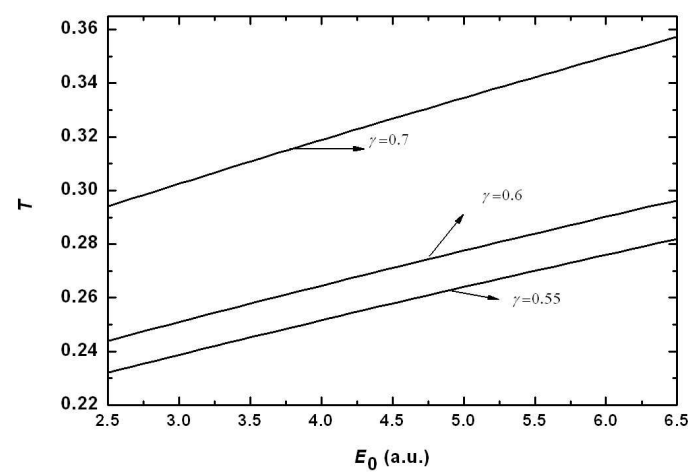

Figure 1: The relational curve of the ground state lifetime of polaron as a function of the ground state energy under different temperature parameter $\gamma$.

Fig. 2 shows the ground-state lifetime as a function of the transverse and longitudinal confinement lengths, with $\alpha=6,6.5,6.7$ and $\gamma=0.6$. From Fig. 2 , it is obvious that the groundstate lifetime decreases with the increasing of transverse and longitudinal confinement lengths of quantum dot. The reason is that there are confinement potential to confine the motion of the electrons. When the confining potential decreases ( $\omega_{1}$ and $\omega_{2}$ decrease), that is, $\rho$ and $z$ increase, the thermal motion energy of the electron and the interaction between the electron and phonons, which take phonon as medium, weaken because the range of the particles motion becomes large, which lead to the ground-state energy decreasing. As a result, the transiton probability per second becomes fast and leads to the reduction of the ground-state lifetime.
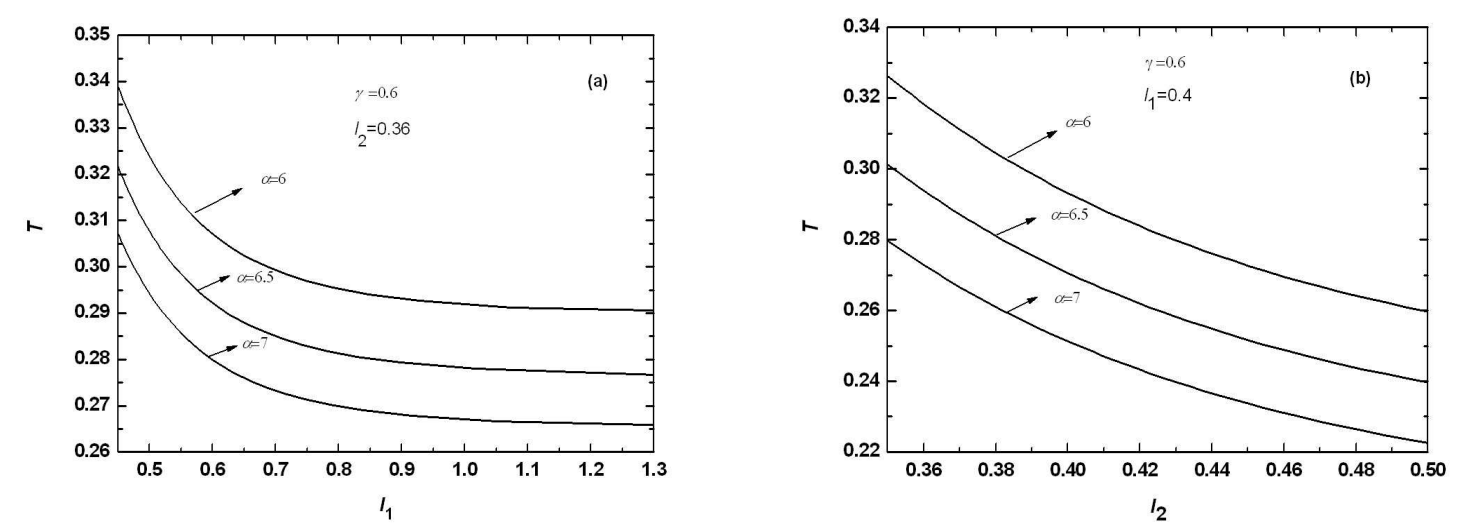

Figure 2: The relational curves of the ground state lifetime of polaron as a function of the transverse confinement length (a) and the longitudinal confinement length (b) under different coupling-strengt $\alpha$.

Fig. 3 indicates the the ground-state lifetime of polaron as a function of the coupledstrength, at $l_{1}=0.4, l_{2}=0.4,0.5,0.8$ and $\gamma=0.6$. It can be seen that the ground-state lifetime of polaron decreases with increasing the coupled-strength. That is because the contribution of the interaction energy between electron and phonons to the ground-state energy is a negative 
value, when the coupled-strength increases, the ground-state energy gradually decreasing. So the transiton probability per second is enlarged, and thus lead to the decrease of the groundstate lifetime.

Fig. 4 shows the relationship between the ground-state lifetime of polaron and the temperature parameter, with $\alpha=5,6,7$. It is easily find from Fig. 4 that the ground-state lifetime of polaron rapidly increases with increasing the temperature parameter. The cause is the lattice thermal motion become slow with the increasing temperature parameter, that is the falling external temperature make the number of phonons around the electron to decrease sharply. As a result, the probability of an electron absorbing a phonon becomes small.

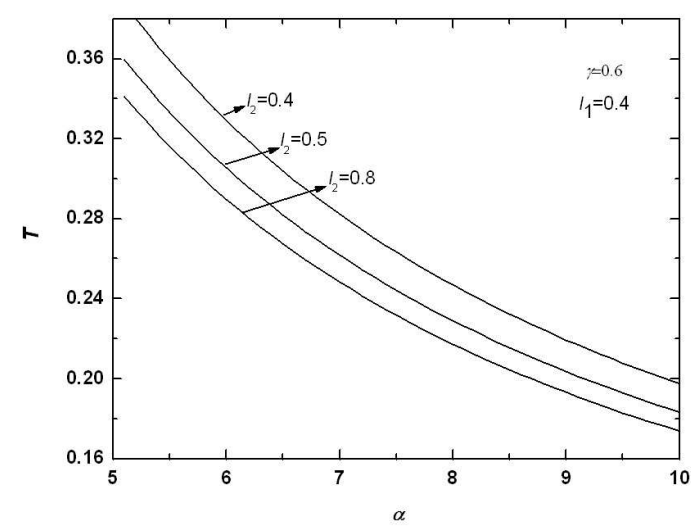

Figure 3: The relational curve of the ground state lifetime of polaron as a function of the coupling strength $\alpha$ under different longitudinal confinement length of quantum dot.

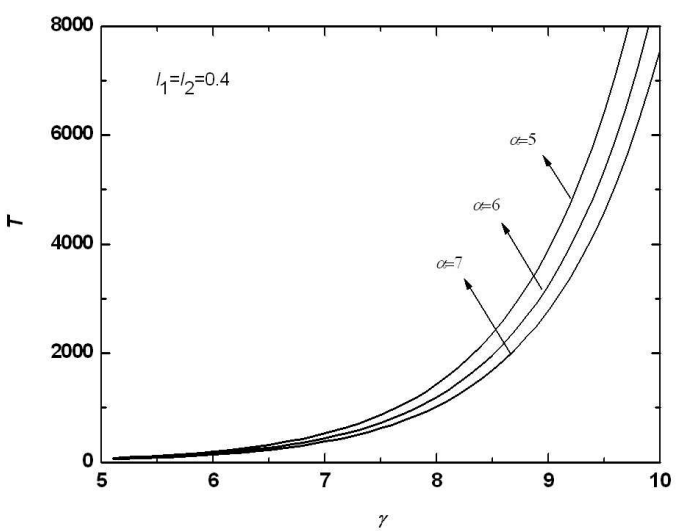

Figure 4: The relational curve of the ground state lifetime of polaron as a function of the temperature parameter $\gamma$ under different coupling-strength $\alpha$. 


\section{Conclusions}

The properties of strong-coupled polaron are researched by using linear combination operator and unitary transformation methods in an asymmetric quantum dot. The ground-state lifetime as a function of the ground-state energy, the electron-phonon coupled-strength and the transverse and longitudinal confinement lengths of quantum dot are obtained. The conclusions are that the ground-state lifetime increases with increasing the ground-state energy, and decreases with increasing the electron-phonon coupled-strength. It is also observed that the ground-state lifetime decreases with increasing the transverse and longitudinal confinement lengths of quantum dot, and rapidly increases with increasing the temperature paramet.

\section{References}

[1] C. Sikorski and U. Merkt, Phys. Rev. Lett. 62 (1989) 2164.

[2] A. Lorke, J. P. Kotthaus, and K. Ploog, Phys. Rev. Lett. 64 (1990 )2559.

[3] S. Normura and T. Kobayashi, Phys. Rev. B 45 (1992) 1305.

[4] S. S. Li and J. B. Xia, Chinese Phys.16 (2007) 1.

[5] S. S. Li and J. B. Xia, J. Appl. Phys.100 (2006) 083714.

[6] S. S. Li and J. B. Xia, Chinese Phys. Lett. 23 (2006) 1896.

[7] S. S. Li, K. Chang, and J. B.Xia, Phys. Rev. B 71 (2005) 155301.

[8] S. D. Sarma, R. D. Sousa, X. D. Hu, and B. Koiller, Solid State Commun.133 (2005) 737.

[9] S. S. Li, J. B. Xia, F. H. Yang, et al., J. Appl. Phys. 90 (2001) 6151.

[10] H. J. Xie and C. Y. Chen, Eur. Phys. J. B 5 (1998) 215.

[11] T. C. Au-Yeung, S. L. Kho, S. W. Gu, and L. H. Hong, J. Phys.: Condens. Matter 12 (2000) 4817.

[12] Y. H. Ren, Q. H. Chen, and Z. K. Jiao, Acta Phys. Sin. 7 (1998) 598.

[13] Q. H. Chen, Z. B. Wang, F. L. Wu, et al., Chinese Phys. Lett. 18 (2001) 668.

[14] S. H. Chen and J. L. Xiao, Chinese J. Semiconductors 27 (2006) 1923.

[15] R. Q. Wang, H. J. Xie, and Y. B.Yu, Phys. Stat. Sol. B 242 (2005) 890.

[16] B. S. Kandemir and A. Cetin, Phys. Rev. B 65 (2002) 054303.

[17] P. Boucaud, S. Sauvage, F. Bras, and G. Fishman, Physica E 26 (2005) 59.

[18] E. A. Zibik, L. R. Wilson, R. P. Green, et al., Physica E 21 (2004) 405.

[19] W. J. Huybrechts, J. Phys. C: Solid State Phys. 10 (1977) 3761. 